\title{
El costo de la salud en adultos mayores: Un estudio descriptivo y retrospectivo en Ecuador
}

Gabith M. Quispe-Fernández ${ }^{1}$, Dante Ayaviri-Nina ${ }^{1}$, Pablo Djabayan-Djibeyan ${ }^{2 *}$ y Otto Arellano-Cepeda ${ }^{1}$

(1) Facultad de Ciencias Políticas y Administrativas, Universidad Nacional de Chimborazo (UNACH), Ave Antonio José de Sucre Km 1 1⁄2 vía Guano, Riobamba, Ecuador (Correo-e: gquispe@unach.edu.ec; dayaviri@unach.edu.ec; oarellano@unach.edu.ec)

(2) Facultad de Ciencias de la Salud - Universidad Nacional de Chimborazo (UNACH), Ave Antonio José de Sucre Km 1 1⁄2 vía Guano, Riobamba, Ecuador (Correo-e: pdjabayan@unach.edu.ec pdjabayan@gmail.com)

* Autor a quien debe ser enviada la correspondencia.

Recibido Feb. 25, 2021; Aceptado Abr. 22, 2021; Versión final Jun. 11, 2021, Publicado Oct. 2021

\section{Resumen}

El objetivo de este estudio descriptivo-retrospectivo fue determinar el costo en salud de los adultos mayores de 60 años en Ecuador desde la perspectiva del paciente. Se consideró una muestra de 5.235 adultos de un total de 1.716.002 adultos. El índice KMO (Kaiser-Meyer-Olkin) cualitativo fue 97,2 \% y el cuantitativo fue 70 $\%$. Los resultados muestran que el costo incluyó consulta médica, hospitalización, pruebas de laboratorio y medicación. El costo promedio total fue de USD 1.833,96/mes y USD 22.007,54/año para el modelo-1 con hospitalización. Para el modelo-2 sin hospitalización, el costo total fue de USD 190,39/mes y USD 2.284,68/año. Se determinó una relación significativa entre costos y uso de servicios de salud en la atención pública y privada. Los costos de salud influyen en el empobrecimiento cuando los ancianos requieren hospitalización. Se concluye que el costo de la polifarmacia es significativamente alto, seguido de la hospitalización en relación con consultas médicas y pruebas de laboratorio.

\section{The cost of health care for older adults: a descriptive and retrospective study in Ecuador}

\begin{abstract}
The main objective of this descriptive-retrospective study was to determine the health costs for adults over 60 years of age in Ecuador from the patient's perspective. A sample of 5,235 adults from 1,716,002 adults was examined. The qualitative and quantitative KMO (Kaiser-Meyer-Olkin) indexes were $97.2 \%$ and $70 \%$ respectively. The results show that the costs included medical consultation, hospitalization, laboratory tests, and medication. The total average cost was USD 1,833.96/month and USD 22,007.54/year for model-1 with hospitalization. The total average cost for model-2 without hospitalization was USD 190.39/month and USD $2,284.68 /$ year. A significant relationship between the cost and the use of health services in public and private care was observed. In addition, health costs influence impoverishment when the elderly require hospitalization. It is concluded that the cost of poly-pharmacy is significantly high, followed by hospitalization costs associated with medical consultations and laboratory tests.
\end{abstract}

Keywords: cost; health; elderly; polypharmacy; hospitalization 


\section{INTRODUCCIÓN}

En Ecuador, como en el resto de países de América Latina y el Caribe, existe un proceso de transición demográfica, que consiste en la reducción de la fecundidad y la mortalidad, consecuentemente, el aumento de la longevidad que impacta la estructura del sistema demográfico y conduce a su virtualización, que está en proceso de revertir la pirámide tradicional con una reducción significativa en la base, constituida fundamentalmente por niños y adolescentes, y un aumento en la cima, conformada por ancianos. Este fenómeno demográfico se da por dos condiciones: en primer lugar, por un aumento del envejecimiento individual, como consecuencia de la reducción de las tasas de mortalidad en edades tempranas y la prolongación de los años de vida como consecuencia del avance de la medicina y la calidad de vida; en segundo lugar, a una disminución de la natalidad, que genera una situación excepcional para las sociedades y estados de la región, que debe ser adecuadamente atendida, para hacer frente a las crecientes demandas de la creciente población de adultos mayores, para lo cual se requiere un cambio en los paradigmas económicos, legales, institucionales y culturales.

La División de Población del Centro Latinoamericano y Caribeño de Demografía (CELADE) que pertenece a la Comisión Económica para América Latina y el Caribe (CEPAL) en su boletín denominado "Datos e Indicadores de Adultos Mayores en América Latina y el Caribe" indicó que en todos los países de América Latina y el Caribe existe un incremento sostenido que implica la población mayor de 60 años debido a un proceso generalizado de longevidad de las estructuras demográficas y con ello, un aumento en el peso específico que tiene este grupo de edad con relación al número total de población. Se indicó que el número de adultos mayores para el año 2000 fue de 41,3 millones de personas ( $8 \%$ de la población total) y que sería de 98,3 millones para el año 2025 (14,1\% de la población total) y 184,3 millones para el año 2050 (22,6 \% de la población total). Este aumento de la longevidad se está produciendo de manera acelerada, en comparación con la velocidad de este aumento en los países desarrollados, ocurriendo a una tasa del 3,5\% para el período 2000-2025, tres veces más rápido que la tasa de crecimiento de la población total, aunque algunos los países de la región crecerán a un ritmo mayor (CELADE, 2002).

Esta creciente longevidad se está dando en la región en un contexto de pobreza, aguda desigualdad de ingresos, escaso desarrollo institucional y persistente desigualdad social. La incidencia de la pobreza entre los ancianos en la mayoría de los países es alta, con cifras que oscilan entre el 30 y $70 \%$, principalmente en las zonas urbanas, aunque en algunos países las tasas más altas se dan en las zonas rurales. El escaso desarrollo institucional y la persistente desigualdad social se reflejan en la baja cobertura de los sistemas de previsión social de pensiones y jubilaciones en la región, a lo que se suma un ingreso mensual insuficiente en la mayoría de los países, que no alcanza para cubrir las necesidades básicas de una pareja. Entre el 25 y $50 \%$ de las personas mayores en el área urbana de la mayoría de los países reciben prestaciones de seguridad social, mientras que solo el $38 \%$ son beneficiarios en el mejor de los casos en el área rural, sin embargo, en algunos países esta cifra desciende al $10 \%$. Esto hace que los servicios de la seguridad social puedan considerarse como un privilegio, ya que en la mayoría de los países se excluye a una parte importante de la población de adultos mayores, particularmente los más pobres, sin embargo, esta desigualdad es menor en algunos países. Es por ello por lo que el $80 \%$ de los adultos mayores viven en dependencia en hogares multigeneracionales, incluso en muchos casos son jefes de hogar, y esta convivencia intergeneracional permite una reducción de los gastos familiares por persona, promueve la economía de escala en la compra y preparación de alimentos y apoya directamente a los miembros de la familia con necesidades especiales (CELADE, 2002).

Las proyecciones realizadas por Miller y Mejía-Guevara (2020) muestra que en Ecuador la población adulta mayor a 60 años va en incremento desde $11 \%$ que es a la fecha hasta un $21 \%$ para el año 2065, llegando a constituir la tercera parte de la población para el 2100, con una población estimada de 9 millones de ecuatorianos adultos. En cambio, la población menor a 60 años sufrirá una disminución a un $40 \%$ llegando a su tope de crecimiento en la población entre los 20 a 59 años hasta el año 2032 convirtiéndose en una sociedad envejecida para el 2065 incrementando enfermedades y su costo y gasto en salud.

De este modo la investigación partió preguntado ¿A cuánto asciende el costo de salud de los adultos mayores? ¿Cuál fue el efecto económico? y ¿Qué repercusiones tuvo? porque no se pudo identificar estudios relacionados al análisis y cálculo de los costos desde la perspectiva del paciente en el Ecuador. Entendiendo que los costos o coste es el gasto económico que representa la fabricación de un producto o la prestación de un servicio, y los costos de salud se refieren a los gastos relacionados a la producción del servicio, entre ellos el pago por prestaciones e insumos. Desde la perspectiva del médico y del paciente, el costo se define como "dolor, sufrimiento, muerte o pérdida de la productividad y beneficio en la exactitud diagnosticada, reducir el sufrimiento o la cura [...] el costo total para la sociedad debe integrar todas estas fuentes para poder realizar una evaluación imparcial de una relación costo vs beneficio" (Cortés-Buelvas et al., 2002). Así, los costos se relacionan al proceso de salud-enfermedad y atención, donde el costo significa un desembolso pasado, 
presente o futuro que implica un beneficio y el gasto un desembolso cuyo valor se consume (MarulandaCastaño, 2009).

Por otro lado, estudiar a los adultos mayores es de suma importancia porque se consideran "un grupo prioritario que se requiere mantener un autocuidado eficiente" (Vargas-Aguilar et al., 2020), además de acuerdo con la Organización Mundial de la Salud, recomienda que los "adultos mayores deben acudir al centro de salud mensualmente o varias veces al año, con el objetivo de prevenir y evitar riesgos de adquirir enfermedades infecciosas o no infecciosas. Estas consultas permiten identificar los factores de riesgo que, al no ser controlados adecuada u oportunamente, pueden transformarse en agentes que, asociados a las limitaciones físicas y emocionales del adulto mayor, pueden causar afecciones leves o severas en las personas mayores de 64 años, y en casos graves tienen la capacidad de incrementar la tasa de defunción" (Vargas-Aguilar et al., 2020). De este modo el objetivo de este estudio descriptivo y retrospectivo fue determinar el costo en salud de los adultos mayores de 60 años en Ecuador desde la perspectiva del paciente, utilizando datos de la última Encuesta de Salud, Bienestar y Envejecimiento (SABE).

Entre los antecedentes se encuentran estudios realizados en Ecuador que indicaron que la percepción de bienestar subjetivo en adultos mayores tuvo como predictor importante a la alimentación que fue relacionada con los recursos percibidos, la situación económica y la percepción de salud, revelando los resultados que los adultos mayores de las zonas urbanas relacionan su bienestar a una percepción mala de salud mientras que en las zonas rurales la calidad de vida se relaciona con una situación económica desfavorable (ArévaloAvecillas et al., 2019), por otro lado, el análisis hecho de los principales determinantes socioeconómicos que afectan la calidad de vida de los adultos mayores concluye que el área de residencia no es determinante, no así la ocupación y el nivel de educación, y un impacto negativo relaciona con el padecimiento de una enfermedad y pertenecer al rango de edad de 71 y 80 años (Bustamante et al., 2017).

La hipoacusia bilateral es una de las alteraciones físicas ocasionada por el envejecimiento, esta condición tiene un impacto negativo en la calidad de vida de los adultos mayores tanto física como psicológicamente, por ello en Chile a través del programa de Garantías Explicitas en Salud (GES) se hace entrega de audífonos a la población adulta mayor, el impacto y el grado de satisfacción por el uso de estos audífonos fue determinado por Bustamante et al. estableciendo que los beneficiarios reconocen su utilidad y los cambios positivos en sus rutinas diarias, reduciendo la posibilidad de caídas y concluyendo que hay una relación directa entre el uso de los audífonos y la calidad de vida de adultos mayores en Chile que los usan (Bustamante et al., 2014a; Bustamante et al., 2014b).

Entre la comorbilidades y complicaciones de salud, la demencia se debe tomar en cuenta al momento de establecer los costos de salud en adultos mayores, esta afirmación deriva de la conclusión obtenida por Bail et al., (2018) mediante la cual afirmaron que las complicaciones y la demencia suponen un mayor costo que otro tipos de complejidades en pacientes adultos mayores hospitalizados; está afirmación ha sido respaldada mediante un estudio realizado en Inglaterra por Wittenberg et al., (2020) en el cual proyectaron que para el año 2040 el número de personas mayores con demencia aumentará a más del doble en un 124\%, de 530.000 a 1.183.000 adultos mayores, este aumento supondría un costo de 80.100 millones de libras esterlinas (£), con un costo promedio anual por persona de $58.900 £$. Por otra parte, ha sido reportado que las caídas en adultos mayores generan costos médicos que deben ser estimados para establecer la magnitud de estos y su efecto financiero en la eficacia de las estrategias de prevención (Florence et al., 2018).

Casas-Vásquez et al. (2016) reportaron que los adultos mayores utilizan más del $50 \%$ de los medicamentos que se expenden, el $80 \%$ consume al menos un medicamento al día y $75 \%$ no informa sobre el uso de tratamientos no convencionales, así mismo, indicaron que la prescripción de medicamentos a los adultos mayores requiere de un balance entre riesgos y beneficios que hace que esta práctica médica sea compleja y difícil, por ello, recomiendan la aplicación de directrices y recomendaciones generales para el manejo farmacológico de la enfermedades crónicas de los adultos mayores y con ello disminuir la medicación inapropiada, los elevados costos de la polifarmacia y la automedicación.

Si se compara la realidad de los países latinoamericanos con la realidad de los países de la Unión Europea, las realidades coinciden en que el envejecimiento de la población no tiene precedente histórico y, en ambos casos, implica nuevos desafíos para los sistemas de salud y seguridad social para garantizar el bienestar de la población de adultos mayores, independientemente de la comprensión de la morbilidad en este grupo. El número de ancianos con: cáncer, fractura de cadera, ictus, cardiopatías, enfermedades articulares, diabetes, hipertensión, menopausia-andropausia y depresión-demencia aumentará en ambas realidades con tendencia a la multimorbilidad; se diferencia con los europeos, porque el aumento proyectado de los gastos en salud como consecuencia del envejecimiento es bajo, mientras que en los países de América Latina es lo inverso; por lo tanto, en ambos casos, se deben buscar las mejores opciones disponibles para adaptar la atención 
médica y los sistemas de seguridad social para satisfacer las necesidades de la población que envejece (Rechel et al., 2013).

Sin embargo, Coory afirma que las predicciones realizadas, marcadas por posiciones ideológicas en la política sanitaria, que indican que el envejecimiento de la población se traducirá en un vertiginoso aumento de los costes sanitarios no son correctas, hay estudios que han demostrado que el efecto del envejecimiento de la población en el gasto en salud es pequeño y manejable. Estas predicciones buscan favorecer un crecimiento del sector privado de salud conteniendo el gasto en el sector público, desviando el foco de atención hacia la evaluación de relevancia y efectividad de los patrones de atención actuales; con esto se podría tener una visión más realista sobre el tema (Coory, 2004). Este tema controvertido conduce ineludiblemente a los gobiernos, sus servicios de salud y la seguridad social, a considerar el hecho incuestionable de que la población anciana está aumentando en todas partes del mundo, por lo tanto, la planificación presupuestaria del gasto público y privado en salud debe realizarse lo más precisa posible en función de la realidad de cada país.

Es por eso por lo que Caley y Sidhu (2010) concluyen que es importante poder predecir con precisión la demanda y los costos de la atención médica dentro del Servicio Nacional de Salud del Reino Unido, lo que no puede subestimarse. Indican que hacer supuestos simplistas y no utilizar principios bien establecidos en los modelos estadísticos utilizados, conduce a resultados muy diferentes que tienen el potencial de tener consecuencias organizacionales negativas y masivas en términos de planificación estratégica de corto a mediano plazo. Existen numerosos estudios en la literatura científica que abordan el tema del costo de los sistemas de atención en salud y seguridad social para la población anciana, que, como se mencionó anteriormente, aumenta cada año. Katon et al., reportaron un incremento en los costos de hospitalización y ambulatorios en una muestra poblacional de pacientes ancianos con diagnóstico clínico depresivo, los cuales fueron entre un 47 a $51 \%$ más altos en comparación con los pacientes de ancianos sin depresión, lo que significó un aumento en los costos ambulatorios y hospitalarios de 1.045 a 1.700 USD por paciente (Katon et al., 2013).

El costo total invertido en 2016 en pacientes con demencia que viven permanentemente en 17 residencias en Australia se calculó a partir de los datos recopilados de 541 de estas personas, con un costo anual total de aproximadamente 72.044 USD, el costo anual promedio en atención residencial fue de 67.000 USD, este costo residencial representó el $93 \%$ del costo total, discriminado de la siguiente manera: $48 \%$ (34.581 USD) en ingreso hospitalario, $31 \%$ (22.334 USD) en productos farmacéuticos y $15 \%$ (10.807 USD) en asistencia extra hospitalaria. Esta estimación indica el alto costo asociado con el cuidado de personas con demencia que vivían permanentemente en residencias de cuidados (Gnanamanickam et al., 2018).

Un estudio retrospectivo realizado en Ontario Canadá, que evaluó el efecto que los factores sociodemográficos tienen sobre la asociación entre la multimorbilidad y el costo atribuible en la atención médica, reveló que el costo total atribuible a la multimorbilidad en individuos jóvenes (<65 años) estuvo entre 377 y 2.073 \$C, mientras que en adultos mayores (> 65 años) estuvo entre 1.026 y 3.831 \$C, también indicó que la asociación entre el grado de multimorbilidad y los costos en la atención de salud se modificó significativamente por edad $(p<0,001)$ y por sexo $(p<0,001)$, siendo mayor en el grupo de género femenino de adultos mayores de 65 años (Thavorn et al., 2017).

Con el objetivo de estimar la prevalencia futura de diabetes en mexicanos mayores de 50 años, evaluando la salud actual y futura, así como la carga económica que la prevalencia de diabetes tendría sobre el sistema de salud, González-González et al., realizaron un estudio de simulación que utilizó datos longitudinales en tres años diferentes, obteniendo que la incidencia de diabetes ha aumentado en la población estudiada con valores que van desde el 4,3 \% entre 2001 y 2003 hasta el 19,3\% en 2012 (3,99 millones de personas) . Los autores también indican que, en un escenario de no intervención, se espera que la prevalencia de diabetes aumente del 19,3\% en 2012 al 34,0 \% en 2050 y con una intervención del $30 \%$ la prevalencia sería del 28,6 $\%$ para 2050, lo que subraya la importancia de la diabetes como enfermedad y la potencial demanda de atención médica y su carga social sobre los costos que requerirán intervenciones y políticas para reducir su incidencia (González-González et al., 2017).

Se espera que la incidencia de cáncer en adultos mayores de 65 años esté aumentando a nivel mundial y, por supuesto, tendrá impactos económicos y sociales globales, este hecho representa un desafío considerable y muy particular para los sistemas de asistencia sanitaria y social de cualquier país, particularmente aquellos con sistemas sociales y de salud débiles y con recursos económicos limitados, para Pilleron et al., la incidencia de cáncer para el 2035, calculada por proyecciones de población, revela que aproximadamente 14 millones de personas serían diagnosticadas con cáncer, casi un $60 \%$ de la incidencia global de cáncer, significativamente mayor en comparación con los valores de incidencia diagnosticados en 2012, que fue de 6,7 millones de ancianos, que representa el 47,5\% del diagnóstico global de cáncer y reportó 
que cerca del $48 \%$ de estos nuevos casos ocurrieron en regiones menos desarrolladas del mundo, siendo el cáncer más frecuente de: pulmón, colorrectal, próstata, estómago y mama, representando $55 \%$ de la incidencia mundial; para 2035, estas regiones menos desarrolladas del planeta verán un aumento de nuevos casos del orden del $144 \%$ en comparación con el $54 \%$ que se espera que ocurra en las regiones desarrolladas (Pilleron et al., 2018).

En una encuesta longitudinal aplicada a jubilados mayores de 45 años diagnosticados de enfermedades cardiovasculares, Li et al., estudiaron, en China continental, la carga financiera para los chinos de mediana edad y mayores con la prevalencia de estas enfermedades y su asociación con el nivel socioeconómico, los resultados revelaron que la ocurrencia de gastos catastróficos en salud de los hogares aumentó de 44,2 \% en 2011 a 48,1 \% en 2013 , con un costo anual por persona para tratar estas enfermedades en 2011 de 770 USD y en 2013 de 970 USD, un aumento del $26 \%$ en el gasto, que consume más del $40 \%$ del presupuesto familiar no alimentario, los pacientes gastaron el doble de lo que se gasta tanto en los servicios ambulatorios como hospitalarios y dos de los tres indicadores de nivel socioeconómico (gasto total del hogar y tipo de ocupación) están asociados al pago individual por la incidencia de enfermedades cardiovasculares y al gasto catastrófico en salud del hogar, además, los desempleados tienen más probabilidades de hacer estos gastos que los trabajadores agrícolas. Concluyen afirmando que el sistema de salud chino debe utilizar este patrón de gasto en salud causado por pacientes con enfermedades cardiovasculares para crear un esquema de seguro de salud más equitativo que establezca un equilibrio en términos de atención ambulatoria y hospitalaria para brindar una mejor protección financiera de los riesgos a los pacientes con bajo nivel socioeconómico (Li et al., 2018).

\section{METODOLOGÍA}

El método aplicado fue descriptivo - retrospectivo. Se informó que la población total de Ecuador para el año 2017 fue de 16.776.977 habitantes y las personas mayores de 60 años fueron 1.716 .002 adultos. Se consideró una muestra de 5.235 ancianos mayores de 60 años, con un nivel de confianza de $95 \%$ y un margen de error de $1,3524 \%$, distribuidos en zonas urbanas y rurales y sean residentes habituales. Se aplicó la entrevista y el índice $\mathrm{KMO}$ en datos cualitativos fue del 97,2 \% y del $70 \%$ en datos cuantitativos, siendo válidos y confiables los datos que corresponden a la última Encuesta de Salud, Bienestar y Envejecimiento de Ecuador (SABE, 2017) y que fueron considerados en el estudio. Para el análisis se utilizó estadísticas descriptivas, modelo matemático en el cálculo de costos y la comprobación de la hipótesis a través del modelo de regresión logística multinomial, debido a que es una de las herramientas estadísticas multivariantes más utilizadas en el análisis de investigaciones en el área salud y en variables categóricas.

Para determinar el costo en salud de los adultos mayores de 60 años en Ecuador desde la perspectiva del paciente se planteó cinco hipótesis: $\mathrm{H} 1$ El costo del gasto privado en salud es diferente según el tipo de enfermedad que padecen los adultos mayores; H2 Se suspenden los medicamentos necesarios que mejoran y mantienen a los ancianos como consecuencia de factores relacionados con el costo, disponibilidad de medicamentos, no tener transporte o no querer tomar medicamentos; H3 Los servicios de salud subsidiados no cubren las necesidades requeridas de manera oportuna, lo que hace que los adultos mayores busquen otras alternativas; H4 El costo y la mala atención son elementos que influyen en el uso de los servicios de salud públicos y privados; H5 Los costos de salud se convierten en gastos d empobrecimiento para los adultos mayores.

\section{El modelo para determinar los costos de salud para las personas mayores}

El costo en salud se determinó considerando variables como: consulta médica, hospitalización, pruebas de laboratorio y medicamentos (polifarmacia) con base en propuestas de cálculo del costo realizados por autores, como Santibáñez-Beltrán et al., (2013), Villarreal-Ríos et al., (2017), Sánchez-Román et al., (2012) y Katon et al., (2003) entre otros autores, quienes determinan los costos por tipo de enfermedad, como se presenta en la Tabla 1. Sin embargo, para este estudio se consideró la estimación de los costos en salud de manera global acercándose a la propuesta de Quevedo-Tejero et al., (2011).

En este contexto, el modelo de determinación de costos (variable dependiente) consideró como variables independientes: 1) variables cuantitativas, como a) costos de salud: consulta médica, tratamiento de enfermedades, medicación, laboratorio, exámenes, hospitalización y otros servicios; b) características económicas: ingresos y gastos; y 2) Variables cualitativas, como, a) características demográficas (edad, nivel de educación, lugar de residencia, género, convivencia familiar); b) características de seguridad sanitaria (tipo de seguro social); c) características clínicas (tipo promedio y número de enfermedades); d) características de la polifarmacia y / o farmacología (tipo, promedio y tiempo de uso de medicamentos y servicios de farmacia). 
Tabla 1. Variables consideradas por otros autores en la determinación de costos

\begin{tabular}{|c|c|c|}
\hline Autores & Titulo & Variables \\
\hline $\begin{array}{l}\text { Santibáñez- } \\
\text { Beltrán et al., } \\
\text { ( 2013) }\end{array}$ & $\begin{array}{l}\text { Costo económico de la } \\
\text { polifarmacia en el adulto mayor } \\
\text { en el primer nivel de atención }\end{array}$ & $\begin{array}{l}\text { Variables sociodemográficas (edad, sexo, estado civil, } \\
\text { escolaridad y ocupación), características clínicas (tipo, promedio } \\
\text { y número de enfermedades crónicas degenerativa; tipo y } \\
\text { porcentaje de complicaciones) y fármacos (tipo, número, } \\
\text { promedio y tiempo de uso) y costo de la polifarmacia evaluada en } \\
\text { tres dimensiones: Consulta médica, medicamentos y servicio de } \\
\text { farmacia }\end{array}$ \\
\hline $\begin{array}{l}\text { Villarreal-Ríos } \\
\text { et al., (2018) }\end{array}$ & $\begin{array}{l}\text { Costo de la atención médica en } \\
\text { pacientes con enfermedad } \\
\text { pulmonar obstructiva crónica }\end{array}$ & $\begin{array}{l}\text { Uso de los servicios de salud y del costo unitario para medicina } \\
\text { familiar, neumología, hospitalización y urgencias; para cada uno } \\
\text { de estos servicios se estimaron los costos de medicamentos, } \\
\text { estudios de laboratorio, estudios de imagen, espirometría, } \\
\text { electrocardiograma, consulta y días de estancia }\end{array}$ \\
\hline $\begin{array}{l}\text { Sánchez- } \\
\text { Román et al., } \\
(2012)\end{array}$ & $\begin{array}{l}\text { Costos de atención médica por } \\
\text { cáncer cervicouterino }\end{array}$ & $\begin{array}{l}\text { Costos médicos directos (consultas médicas, exámenes de } \\
\text { imagenología y laboratorio, radioterapia, hospitalización, cirugía, } \\
\text { quimioterapia, medicamentos, interpretación de laminillas, } \\
\text { transfusiones e incapacidades); costos en intervenciones } \\
\text { quirúrgicas; costos de sesiones de quimioterapia; Costos de } \\
\text { transfusiones, costos en medicamentos, costos por } \\
\text { incapacidades. }\end{array}$ \\
\hline $\begin{array}{l}\text { Katon et al., } \\
(2003)\end{array}$ & $\begin{array}{l}\text { Aumento de los costos } \\
\text { médicos de una muestra } \\
\text { poblacional de pacientes } \\
\text { ancianos deprimidos. Archivos } \\
\text { de psiquiatría general }\end{array}$ & Costos de médicos ambulatorios y de hospitalización \\
\hline $\begin{array}{l}\text { Quevedo- } \\
\text { Tejero et al., } \\
(2011)\end{array}$ & $\begin{array}{l}\text { Fractura de cadera en adultos } \\
\text { mayores: prevalencia y costos } \\
\text { en dos hospitales. Tabasco, } \\
\text { México, } 2009\end{array}$ & $\begin{array}{l}\text { Costo de atención medica hospitalaria por paciente, Costos de } \\
\text { hospitalización, Honorarios médicos por día, Laboratorio, } \\
\text { Radiodiagnóstico, Traslado de ambulancia }\end{array}$ \\
\hline
\end{tabular}

Los costos de salud para un adulto mayor se expresan matemáticamente de la siguiente manera: 1) Determinación del costo directo $(\mathrm{C})$ de cada recurso utilizado por el adulto mayor que es igual a la sumatoria del costo unitario del recurso de salud multiplicado por el número de unidades de un recurso de salud utilizado y 2) por la determinación del costo total en salud del anciano (CTSAM) que es la suma de los costos de cada uno de los recursos de salud utilizados.

$$
\begin{aligned}
& (C)=\sum_{i=1}^{n} Q_{i x} P i=\sum_{i=1}^{m} C a \\
& n \\
& (C T S A M)=\sum_{i=1} C a+C b+C c+C d \ldots \ldots C m n
\end{aligned}
$$

Además, se utiliza la relación costo ingreso para demostrar las hipótesis 5 (H5) a partir de la aplicación de dos modelos, donde se utiliza como modelo 1 la variable dependiente: Ingreso y la variable independiente: costo con hospitalización; y en el modelo 2 se considera como variable independiente: costo sin hospitalización.

\section{RESULTADOS}

La caracterización sociodemográfica del adulto mayor en Ecuador se realizó con los datos obtenidos de las encuestas aplicadas por el Instituto Nacional de Estadística y Censos y de la Comisión Económica para América Latina y el Caribe y del Centro Demográfico de América Latina y el Caribe (INEC, 2003; SABE, 2017), los cuales mostraron que existe un crecimiento poblacional de adultos mayores, una reducción de la tasa de fecundidad y un aumento de la esperanza de vida de las personas (Tabla 2) y con ello la aparición de aspectos importantes a considerar.

Los resultados de la última encuesta de salud, bienestar y envejecimiento también mostraron que hay aproximadamente un 10,2\% de la población adulta en el Ecuador (1.716.002 adultos de una población de 16.776.977 habitantes) con tendencia de un incremento al $21 \%$ para el 2050 . La edad de los adultos mayores varía de 60 a 74 años, lo que representa 3,3\% y $5.3 \%$ respectivamente en cuanto a grupos de edad, disminuyendo este porcentaje en el rango de 75 a 102 años de $8 \%$ a $0,1 \%$, la edad promedio fue de 74,41 años. El $55 \%$ de los ancianos vive en el área urbana y el $45 \%$ en el área rural. La evaluación del nivel de 
instrucción reveló que: 77 \% tiene un nivel de educación primaria, 12,5\% secundaria y 4,7\% nivel superior y solo $0,6 \%$ estudios de posgrado. El género de los ancianos fue $44,9 \%$ hombres y $55,1 \%$ mujeres. Asimismo, el estudio reveló que el 89,5 \% vivía acompañado y el 10,5\% vivía solo; de los que viven acompañados, el $1,20 \%$ vivía con su cónyuge, el 31,52 \% vivía con sus hijos casados, el 14,19 \% vivía con hijos solteros, el $37,88 \%$ vivía con otros familiares y el $15,21 \%$ vivía con otros no familiares. Las razones para convivir con los hijos reflejan que el $28,8 \%$ se debe a problemas de salud personales, el $6,8 \%$ a problemas de salud de los hijos o familiares y el $16,1 \%$ a problemas económicos personales.

Tabla 2. Caracterización sociodemográfica del adulto mayor (Datos tomados de INEC, 2003; SABE, 2017).

\begin{tabular}{|c|c|c|c|c|c|c|c|}
\hline \multicolumn{2}{|c|}{$\begin{array}{c}\text { Tasa de crecimiento } \\
\text { natural }\end{array}$} & \multicolumn{2}{c|}{$\begin{array}{c}\text { Tasa global de } \\
\text { fecundidad }\end{array}$} & \multicolumn{2}{c|}{$\begin{array}{c}\text { La esperanza de vida al } \\
\text { nacer }\end{array}$} & \multicolumn{2}{c|}{$\begin{array}{c}\text { Porcentaje de adultos } \\
\text { mayores }\end{array}$} \\
\hline Período & $\%$ & Período & $\%$ & Período & $\%$ & Período & $\%$ \\
\hline $1985-90$ & 2,42 & $1985-90$ & 4,0 & $1985-90$ & 67,5 & 1985 & 4,0 \\
\hline $2005-10$ & 1,62 & $2005-2010$ & 2,6 & $2005-2010$ & 75,0 & 2005 & 5,7 \\
\hline 2016 & 1,31 & $2010-2015$ & 2,4 & $2010-2015$ & 75,9 & 2016 & 9,3 \\
\hline & & $2015-2020$ & 2,2 & $2015-2020$ & 76,8 & 2017 & 10,2 \\
\hline & & $2020-2025$ & 2,1 & $2020-2025$ & 77,3 & & \\
\hline
\end{tabular}

Fuentes de ingresos y seguros de salud de las personas mayores en Ecuador.

Las fuentes de financiamiento provienen de: actividad laboral (59\%), jubilación (17\%), familiares de otros países $(7 \%)$, familiares dentro del país $(2 \%)$, ingresos o ingresos bancarios $(6 \%)$ y otras fuentes $(9 \%)$. Mientras que el $30,4 \%$ cuenta con los siguientes seguros médicos: seguro general $16,2 \%$; seguro campesino $9,2 \%$; seguro privado $2 \%$; seguro policial 1,8\%; seguro municipal $1,2 \%$.

Patologías más comunes del adulto mayor en Ecuador.

De acuerdo con la Constitución Política de la República del Ecuador en su artículo 36, menciona que "las personas mayores recibirán atención prioritaria y especializada en el ámbito público y privado, especialmente en los campos de inclusión social y económica, y protección contra la violencia" (Constitución de la República del Ecuador 2008). Los resultados mostraron que las personas mayores presentan diferentes patologías que requieren consulta, tratamiento, medicación y otros servicios de salud que son necesarios para una adecuada recuperación, como patologías relacionadas con: problemas articulares $(63,7 \%)$, dolor de espalda (49,8 \%), fatiga severa o cansancio $(47,1 \%)$ y patologías diagnosticadas por el médico: hipertensión arterial $(43,7 \%)$, artritis o reumatismo $(31,5 \%)$, osteoporosis $(17,3 \%)$, diabetes $(12,6 \%)$ y cardiopatías $(12,2 \%)$, entre otros, como se muestra en la Tabla 3. Asimismo, las patologías y fármacos más prevalentes se relacionaron con: $47,7 \%$ corazón ( $5,8 \%$ s / total), $28 \%$ cerebro (1,7\% s / total), $22,9 \%$ articulaciones (10,7 \% s / total), 57,9 $\%$ presión arterial $(25,3 \% \mathrm{~s} /$ total), $63,3 \%$ diabetes ( $8 \% \mathrm{~s} /$ total), $10,3 \%$ menopausia $(5,5 \% \mathrm{~s} /$ total) y 31 $\%$ depresión (3,6\% s/ total).

\section{Determinación de los costos de los servicios de salud para la población anciana}

Los costos de los servicios de salud son importantes para determinar los efectos sobre la salud de los ancianos, en este contexto los resultados de la investigación mostraron que, los costos promedio de las consultas médicas alcanzaron los 9,75 USD (entre USD 5 y 100) / Mes y USD 117,00 / Año; mientras que la hospitalización alcanzó los 1.643,57 USD / Mes (mínimo 200 y máximo 50.000 USD por un promedio de 14 noches) y 19.722,86 USD / Año, los exámenes de laboratorio llegaron a 142,50 USD (entre 50 y 2.000 USD) / Mes y 1.710,00 USD / Año y medicamentos 38,14 USD (entre 50 y 2.000 USD / mes, número de medicamentos promedio utilizados 3,74 veces al día) y 457,68 / año. El costo total promedio es de 1.833,96 USD por mes y 22.007,54 por año, considerando los costos de hospitalización, donde al menos en promedio fue hospitalizado una vez cada tres meses durante 14 días (total $5 \%$ de los ancianos), dentro de un parámetro de 1 a 60 días. (Modelo 1). Sin embargo, si los ancianos no requirieron hospitalización, el costo total promedio por mes alcanzó los 190,03 USD y 2.284,68 USD por año (Modelo 2), como se muestra en la Tabla 4. Estos costes en el caso de la polifarmacia corresponden al consumo de fármacos del $56,3 \%$ de los adultos mayores, distribuidos en medicamentos relacionados con el cáncer, diabetes, corazón, presión, articulaciones y artritis y cerebro, como se observa en la Tabla 5. 
Tabla 3. Patologías diagnosticadas y relacionadas con diferentes problemas (Datos tomados de SABE, 2017).

\begin{tabular}{|c|c|c|c|c|}
\hline Patologías diagnosticadas por el doctor & Si \% & No $\%$ & No Responde \% & Total \% \\
\hline Presión alta & 43,7 & 54,5 & 1,8 & 100 \\
\hline Diabetes & 12,6 & 85,6 & 1,8 & 100 \\
\hline Cáncer & 2,7 & 96,9 & 0,4 & 100 \\
\hline Pulmonar o crónica & 7,9 & 91,6 & 0,5 & 100 \\
\hline Tuberculosis & 1,3 & 98,1 & 0,6 & 100 \\
\hline Corazón & 12,2 & 87,2 & 0,6 & 100 \\
\hline Derrame cerebral & 6,2 & 93,5 & 0,3 & 100 \\
\hline Artritis, reumatismo & 31,5 & 6,7 & 1,5 & 100 \\
\hline Fracturas & 4,1 & 95,8 & 0,1 & 100 \\
\hline Osteoporosis & 17,3 & 80,6 & 2,1 & 100 \\
\hline Pierde involuntariamente las heces & 6,1 & 93,1 & 0,8 & 100 \\
\hline Patologías relacionadas con & Si\% & No $\%$ & No Responde \% & Total \% \\
\hline Dolor de pecho & 31,2 & 68,5 & 0,3 & 100 \\
\hline Hinchazón en los pies & 33,2 & 66,6 & 0,2 & 100 \\
\hline Falta de aire al respirar & 26,7 & 73,0 & 0,3 & 100 \\
\hline Mareos persistentes & 43,7 & 55,9 & 0,4 & 100 \\
\hline Dolor de espalda & 49,8 & 50,0 & 0,2 & 100 \\
\hline Dolor de cabeza persistente & 40,6 & 59,2 & 0,2 & 100 \\
\hline Fatiga o cansancio severo & 47,1 & 52,6 & 0,3 & 100 \\
\hline Tos persistente & 32,2 & 67,5 & 0,3 & 100 \\
\hline Problemas en articulaciones & 63,7 & 36,1 & 0,2 & 100 \\
\hline Nauseas persistentes & 11,2 & 88,6 & 0,2 & 100 \\
\hline Sudoración excesiva & 25,9 & 73,7 & 0,4 & 100 \\
\hline Diagnóstico de cataratas & 25,8 & 72,4 & 1,8 & 100 \\
\hline Glaucoma & 30,4 & 94,7 & 1,9 & 100 \\
\hline Problemas nerviosos o mentales & 11,3 & 88,1 & 0,6 & 100 \\
\hline
\end{tabular}


Tabla 4. Determinación de costes sanitarios en personas mayores (Datos tomados de la Encuesta SABE, 2017).

\begin{tabular}{|c|c|c|c|c|}
\hline \multicolumn{5}{|c|}{ Modelo 1} \\
\hline Tipo de costo de la salud & $\begin{array}{c}\text { Costo promedio } \\
\text { en USD }\end{array}$ & Tiempo & $\begin{array}{c}\text { Costo mensual } \\
\text { en USD }\end{array}$ & $\begin{array}{c}\text { Costo anual } \\
\text { en USD }\end{array}$ \\
\hline Costos de medicamentos & 152,56 & 4 meses & 38,14 & 457,68 \\
\hline Costos en hospitalización & 767,00 & 14 días & $1.643,57$ & $19.722,86$ \\
\hline Costos en consultas & 39,00 & 4 veces & 9,75 & 117,00 \\
\hline Costos en exámenes de laboratorio & 570,00 & 4 veces & 142,50 & $1.710,00$ \\
\hline Costo total & & & $1.833,96$ & $22.007,54$ \\
\hline \multicolumn{5}{|c|}{ Modelo 2} \\
\hline Tipo de costo de la salud & $\begin{array}{c}\text { Costo promedio } \\
\text { en USD }\end{array}$ & Tiempo & $\begin{array}{c}\text { Costo mensual } \\
\text { en USD }\end{array}$ & $\begin{array}{c}\text { Costo anual } \\
\text { en USD }\end{array}$ \\
\hline Costos de medicamentos & 152,56 & 4 meses & 38,14 & 457,68 \\
\hline Costos en hospitalización & 767,14 & 14 días & 0,00 & 0,00 \\
\hline Costos en consultas & 39,00 & 4 veces & 9,75 & 117,00 \\
\hline Costos en exámenes de laboratorio & 570,00 & 4 veces & 142,50 & $1.710,00$ \\
\hline Costo total & & & 190,39 & $2.284,68$ \\
\hline
\end{tabular}

Tabla 5. Tipo de medicamentos consumidos por diferentes patologías (Datos tomados de SABE 2017).

\begin{tabular}{|l|l|l|c|c|}
\hline \multicolumn{1}{|c|}{ Medicamentos para } & Si \% & No \% & No responde \% & Total \% \\
\hline Presión arterial & 57,9 & 42,0 & 0,1 & 100 \\
\hline Diabetes & 63,3 & 36,6 & 0,1 & 100 \\
\hline Inyecciones diabetes & 15,7 & 84,0 & 0,3 & 100 \\
\hline Tratamiento del cáncer & 69,9 & 30,1 & 0,0 & 100 \\
\hline Cáncer & 28,0 & 72,0 & 0,0 & 100 \\
\hline Enfermedad pulmonar & 27,1 & 72,6 & 0,3 & 100 \\
\hline El corazón & 47,7 & 51,8 & 0,5 & 100 \\
\hline Problemas cerebrales & 28,0 & 71,7 & 0,3 & 100 \\
\hline Artritis y reumatismos & 33,9 & 66,0 & 0,1 & 100 \\
\hline
\end{tabular}

En promedio, los adultos mayores consumen 4 medicamentos; sin embargo, el rango de consumo varía de 1 a 19 tipos de drogas que se pueden consumir. Por tanto, el gasto en medicamentos o polifarmacia de los ancianos osciló entre 50 USD o menos (62,8 \%); más de 51 pero menos de / o 100 USD (10,6 \%); entre 101 pero menos de / o 200 USD (4\%), entre 201 pero menos de 500 USD (1,1\%); más de 501 pero menos de / o 2.000 USD (0,2\%) y sin gastos (12,5\%), siendo en promedio un costo de 152,56 USD. LoS gastos de hospitalización oscilaron entre menos de 200 USD (45,4 \%); de 201 pero menos de / o 500 USD (18,4\%); más de 501 pero menos de / o 1.000 USD (11,3\%); más de 1.001 pero menos de / o 2.000 USD (4,3 \%) y más de 2.001 pero menos de / o 50.000 USD (7,9\%) siendo en promedio un costo de 767,14 USD.

En cuanto a las consultas, los costos oscilaron entre menos de 5 USD (32,2\%); más de 6 pero menos de / o 10 USD (23,5 \%); más de 11 pero menos de 30 USD (28,3\%); más de 31 pero menos de / o 50 USD (5,7\%) y más de 51 pero menos de / o 100 USD (1,7\%); siendo en promedio un costo de 39 USD. Los costos de los exámenes de laboratorio oscilaron entre: menos de 50 USD (69,9\%); más de 51 pero menos de / o 100 USD (12,7 \%); más de 101 pero menos de / o 200 USD (4,6 \%); más de 201 pero menos de / o 500 USD (2,4 \%) 
y más de 501 pero menos de / o 1.000 USD (0,4 \%); siendo en promedio un costo de 570 USD. Demostrando que la hipótesis $\mathrm{H} 1$ = Los costos del gasto privado en salud es diferente según el tipo de enfermedad que padecen los ancianos, es cierta

\section{Aplicación del modelo de regresión logística multinomial - suspensión a la medicación}

Los adultos mayores demostraron que tienen diferentes necesidades de salud en atención médica y medicación, esto significa un aumento de la salud de los ancianos, los resultados mostraron que el 43,7\% deja de consumir lo necesario medicamentos que mejoran y mantienen al anciano y el último año fue de 27,5 $\%$. Los factores que explican la suspensión de la medicación después de aplicar el modelo de regresión logísticas multinomial (el modelo es significante para su aplicación Sig. $=0,00$ ) se relacionan: 1) Costo $19 \%$ $\left(R^{2}=38 \%\right)$; 2) Disponibilidad de medicamentos $\left.1,4 \%\left(R^{2}=5 \%\right) ; 3\right)$ No tener transporte $0,6 \%\left(R^{2}=2,3 \%\right)$; 4) No le gusta tomar medicamentos $2.8 \%\left(R^{2}=1,7 \%\right)$ y; 5$)$ Otras razones $6 \%\left(R^{2}=3,8 \%\right)$ como se presenta en la Tabla 6 contrastando la hipótesis $\mathrm{H} 2$ = Se suspenden los medicamentos necesarios que mejoran y mantienen a los ancianos como consecuencia de factores relacionados con el costo, disponibilidad de medicamentos, no tener transporte o no les gusta tomar medicamentos en un $69,3 \%\left(R^{2}=0,693\right.$; Sig. 0,00), es cierta; siendo la variable con mayor explicación el costo en un $38 \%\left(R^{2}=0,38\right.$; Sig. 0, 00).

Tabla 6. Resultado de regresión logística multinomial (Datos tomados de la Encuesta SABE, 2017).

\begin{tabular}{|c|c|c|c|c|c|c|c|c|}
\hline \multicolumn{6}{|c|}{ Contraste de la razón de verosimilitud } & \multicolumn{3}{|c|}{$\begin{array}{l}\text { pseudo } R \\
\text { cuadrado }\end{array}$} \\
\hline $\begin{array}{c}\text { Efecto } \\
\text { Suspensión de la medicación }\end{array}$ & $\begin{array}{c}\text { Logaritmo } \\
\text { de la } \\
\text { verosimilitud } \\
-2 \text { de } \\
\text { modelo } \\
\text { reducido }\end{array}$ & $\begin{array}{l}\text { Chi- } \\
\text { cuadrado }\end{array}$ & gl & $\begin{array}{c}\text { Sig. } \\
\text { TOTAL } \\
\text { e9_1+e9 } \\
2+\mathrm{e} 9 \text { _3 } \\
\text { +e9_4+e } \\
9 \_5\end{array}$ & $\begin{array}{l}\text { Sig. } \\
\text { PARCI } \\
\text { ALES }\end{array}$ & $\begin{array}{c}\text { Cox } \\
y \\
\text { Snell }\end{array}$ & $\begin{array}{c}\text { Nagel } \\
\text { kerke } \\
\text { R2 }\end{array}$ & $\begin{array}{l}\text { McFa } \\
\text { dden }\end{array}$ \\
\hline Interceptación & $2.821,676^{a}$ & 0,00 & 0 & & & & & \\
\hline e9_1 Dejo de tomar $x$ el costo & $5.962,640^{\mathrm{b}}$ & $3.140,96$ & 3 & 0,00 & 0,00 & 0,27 & 0,38 & 0,26 \\
\hline e9_2 Dejo de tomar $x$ la medicina no está disponible & $2.899,616^{b}$ & 77,94 & 3 & 0,00 & 0,00 & ,003 & ,005 &, 003 \\
\hline e9_3 Dejo de tomar $x$ no tener transporte & $2.827,323^{b}$ & 5,65 & 3 & 0,13 & 0,00 & ,016 & ,023 &, 013 \\
\hline e9_4 Dejo de tomar $x$ no le gusta las medicinas & $3.315,569^{b}$ & $4.93,89$ & 3 & 0,00 & 0,00 & ,012 & ,017 &, 010 \\
\hline e9_5 Dejo de tomar $x$ otras razones & $4.091,436^{b}$ & $1.269,76$ & 3 & 0,00 & 0,00 & ,027 & ,038 & ,022 \\
\hline e9_1+e9_2+e9_3+e9_4+e9_5 & & & & & & ,488 & ,693 &, 549 \\
\hline
\end{tabular}

\section{Relación entre costo y las consultas médicas}

En el caso de las consultas médicas, los adultos mayores acuden al hospital público o seguro privado en un $25,7 \%$, centro de salud del Instituto Ecuatoriano de Seguridad Social (IESS) en 8,6 \%, subcentro del IESS $(9,3 \%)$, centro de salud ( $3,2 \%)$, urgencias del hospital público $(0,8 \%)$, a una clínica de la Sociedad de Lucha contra el Cáncer (SOLCA) en un 4,3\%, a una clínica privada (9,9\%), a una consulta privada (34,9\%), hospital privado $(0,4 \%)$, a boticario o farmacia $(3,4 \%)$, lo que significa que a pesar de contar con los beneficios de la seguridad social para los ancianos, en un gran porcentaje de los adultos mayores acuden a consultorios privados y clínicas privadas a pesar de los altos costos, lo que significa que los gastos no los cubren principalmente los ancianos, sino los miembros de la familia. Por lo tanto, la hipótesis $\mathrm{H} 3=\mathrm{Los}$ servicios de salud subsidiados no cubren los servicios requeridos de manera oportuna, lo que hace que los adultos mayores busquen otras alternativas, es cierta.

Se identificó que el $45,3 \%$ de la población anciana no asiste a una consulta médica debido a varios factores, los resultados de regresión logística multinomial, permite identificar sus causas, como: el costo de la atención médica en $43,7 \%\left(R^{2}=0,437\right.$; sig. 0,000$)$, costo del viaje $12,3 \%\left(R^{2}=0,0,123\right.$; sig. 0,000$)$, tiempo del viaje $11,6 \%\left(R^{2}=0,116\right.$; sig. 0,000$)$, la percepción de que los problemas de salud no fueron graves $12,3 \%$ $\left(R^{2}=0,123\right.$; sig. 0,000$)$, mala atención de la salud $6,9 \%\left(R^{2}=0,069\right.$; sig. 0,000$)$, por motivos familiares 5,8 $\%\left(R^{2}=0,058\right.$; sig. 0,000), automedicación $10.9 \%\left(R^{2}=0,109\right.$; sig. 0,000), malestar propio de la vejes $7,7 \%$ $\left(R^{2}=0,077\right.$; sig. 0,000$)$, otros $16,8 \%\left(R^{2}=0,168 ;\right.$ sig. 0,000$)$ (ver Tabla 7$)$, demostrando que $H 4=$ costo y mala atención son elementos que influyen en el uso de los servicios de salud públicos y privados, es cierta. 
Tabla 7. Contraste de la razón de verosimilitud (Datos tomados de SABE, 2017)

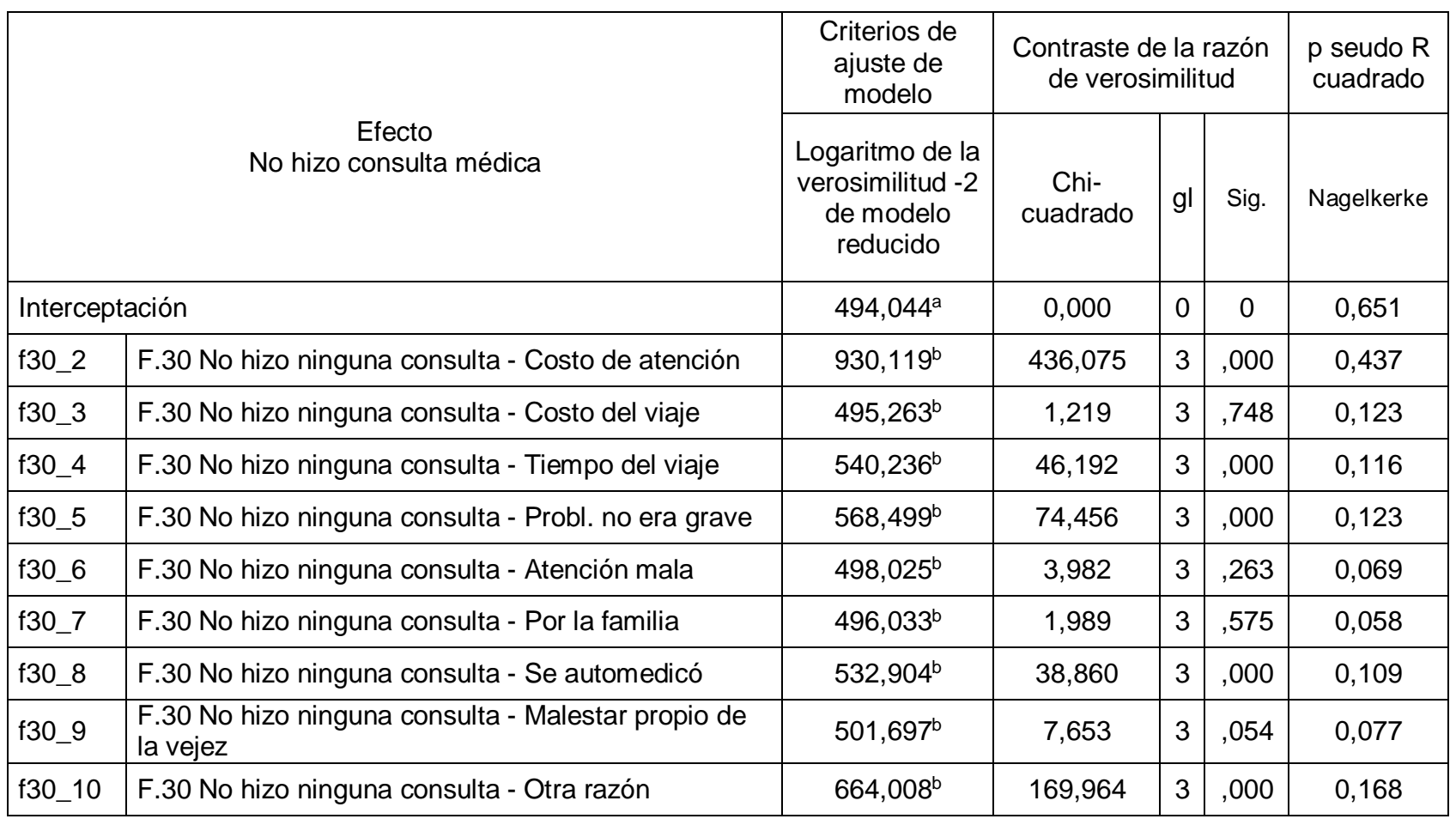

Asimismo, se identificó que el $82,8 \%$ de los ancianos en el período evaluado estaban enfermos, de estos el $67,8 \%$ solicitó atención médica y el $32,1 \%$ no, además las veces que solicitaron atención médica en promedio fue de hasta 3 veces. Los que recibieron atención médica fueron atendidos en un $95 \%$ por un médico, 1,9\% por un curandero, herbolario / masajeador, $1,7 \%$ por un boticario / farmacéutico, 0,7 \% por una enfermera y $0.6 \%$ por otro. Además, los adultos mayores han recibido atención médica en: consultorio privado (36,8 \%), hospitales públicos de: Ministerio de Salud Pública (MSP), IESS, Fuerzas Armadas (FFAA) e Instituto de Seguridad Social de la Policía Nacional (ISSPOL) (22\%). ), subcentro de salud pública MSP / IESS (10,8\%), centro de salud pública MPS / IESS $(6,2 \%)$, hospital o clínica de juntas benéficas (JBG) y Sociedad de lucha contra el cáncer en Ecuador (SOLCA) (5,8\%), droguería o farmacia (5,7\%), centro de salud privado (5,1\%), puesto de salud $(3 \%)$, domicilio $(2,4 \%)$, sala de emergencias en hospitales públicos $(0,6 \%)$, sala de emergencias en hospitales privados $(0,1 \%)$, otros $(1,1 \%)$.

Los factores que explican que los adultos mayores no reciben atención médica están relacionados con factores de disponibilidad de acuerdo con resultados de la aplicación de regresión logística multilineal), como: hospitales, centros de salud, puestos de salud, consultorios, clínicas privadas, naturistas y otros en un $4,8 \%$ $\left(R^{2}=0.048\right.$; Sig. $\left.=0.000\right)$, donde no afecta significativamente la disponibilidad de emergencia en los hospitales públicos y privados, un puesto de salud, farmacias y asilos.

\section{Relación entre gastos de salud e ingresos}

Los resultados de la investigación muestran que el $92,5 \% \mathrm{~s} /$ total recibe ingresos de diferentes medios, de estos, el $60 \%$ de los adultos mayores trabaja y el $40 \%$ no trabaja. Los adultos mayores que trabajan mencionan que los motivos para trabajar son: por necesidad $64,6 \%$, ayudar a su familia $17,3 \%$, mantenerse ocupados $5,5 \%$, sentirse útiles $4 \%$, porque les gusta su trabajo $6,8 \%$, por otras razones, $0,7 \%$ y no sabe $1,1 \%$. Los resultados de la investigación muestran que el ingreso anual promedio expresado en USD que recibe un adulto mayor en el año alcanza los 5.643,74 USD y 470,31 mensuales en promedio, lo que proviene de fuentes como: a) por trabajo que desarrolla 692,88 USD / Mes, por jubilación o pensión 762,13 USD / Mes; por familiares de otros países 191,18 USD / mes; por familiares dentro del país 96,91 USD / Mes; para ingresos o egresos bancarios 1.011,51 USD / Mes; por bono de desarrollo humano 120 USD / mes; otras fuentes 527,58 USD / mes; también ingresos anuales según se especifica en la Tabla 8. Los gastos en que incurren los adultos mayores están relacionados con gastos del hogar, transporte, médicos, vestimenta, alimentación y otros, como se muestra en el Tabla 9. Donde los costos de salud representan un gasto de 19,4 $\%$ s / total de gastos, en relación con los gastos de alimentación de 20,7\% s / total, gastos de vestuario 19,7 $\% \mathrm{~s} /$ total, gastos de transporte $17,2 \% \mathrm{~s} /$ total y gastos de casa $8 \% \mathrm{~s} /$ total. 
Tabla 8. Determinación del ingreso de la población adulta mayor (expresado en USD/promedio) (Datos SABE, 2017).

\begin{tabular}{|l|c|c|c|c|c|c|c|}
\hline \multicolumn{1}{|c|}{ Fuente de ingresos } & $\begin{array}{c}\text { Monto } \\
\text { USD }\end{array}$ & Meses & $\begin{array}{c}\text { No de } \\
\text { veces }\end{array}$ & $\begin{array}{c}\text { Ingreso } \\
\text { mensual } \\
\text { USD }\end{array}$ & $\begin{array}{c}\text { Ingreso } \\
\text { anual } \\
\text { USD }\end{array}$ & $\begin{array}{c}\% \\
\text { Población } \\
\text { s/ 100 }\end{array}$ & $\begin{array}{c}\% \\
\text { Población } \\
\text { s/total }\end{array}$ \\
\hline Ingreso por trabajar & $5.543,01$ & 8 & 1 & 692,88 & $8.314,52$ & 60,00 & 36,72 \\
\hline Ingresos por jubilación o pensión & $2.286,38$ & 3 & 2 & 762,13 & $9.145,52$ & 14,40 & 8,81 \\
\hline $\begin{array}{l}\text { Ingresos por familiares de otros } \\
\text { países }\end{array}$ & 573,53 & 3 & 2 & 191,18 & $2.294,12$ & 12,70 & 7,77 \\
\hline $\begin{array}{l}\text { Ingresos por familiares dentro del } \\
\text { país }\end{array}$ & 290,73 & 3 & 2 & 96,91 & $1.162,92$ & 42,00 & 25,70 \\
\hline $\begin{array}{l}\text { Recibe ingresos por rentas o } \\
\text { ingreso bancarios }\end{array}$ & $1.011,51$ & 1 & 1 & $1.011,51$ & $12.138,12$ & 5,00 & 3,06 \\
\hline $\begin{array}{l}\text { Recibe ingresos por bono de } \\
\text { desarrollo humano }\end{array}$ & 30,00 & 3 & 1 & 10,00 & 120,00 & 26,40 & 16,16 \\
\hline \begin{tabular}{l} 
Recibe ingreso de otras fuentes \\
\hline
\end{tabular} & $1.582,75$ & 3 & 2 & 527,58 & $6.331,00$ & 2,90 & 1,77 \\
\hline Promedio de ingreso & & 470,31 & $5.643,74$ & 163,40 & 100,00 \\
\hline
\end{tabular}

Tabla 9. Gastos incurridos por adultos mayores como porcentaje (Datos tomados de la Encuesta SABE, 2017).

\begin{tabular}{|c|c|c|c|c|c|c|}
\hline Respuestas & $\begin{array}{c}\text { Gastos de la } \\
\text { casa \% }\end{array}$ & $\begin{array}{l}\text { Gastos en } \\
\text { comida \% }\end{array}$ & $\begin{array}{l}\text { Gastos en } \\
\text { ropa \% }\end{array}$ & $\begin{array}{l}\text { Gastos } \\
\text { médicos } \%\end{array}$ & $\begin{array}{c}\text { Gastos de paseos y } \\
\text { transporte } \%\end{array}$ & $\begin{array}{c}\text { Otros } \\
\text { gastos \% }\end{array}$ \\
\hline Si, todo & 13,6 & 28,0 & 34,8 & 33,2 & 35,3 & 26,2 \\
\hline Si, en parte & 9,1 & 38,9 & 24,8 & 27,8 & 20,1 & 18,5 \\
\hline Alguien más & 13,5 & 26,5 & 29,5 & 26,7 & 22,4 & 23,1 \\
\hline $\begin{array}{l}\% \text { de gasto } \\
\text { /total }\end{array}$ & 36,2 & 93,4 & 89,1 & 87,7 & 77,8 & 67,8 \\
\hline No lo hace & 63,6 & 6,6 & 10,9 & 12,1 & 21,9 & 24,5 \\
\hline No responde & 0,2 & 0,0 & 0,0 & 0,2 & 0,3 & 7,7 \\
\hline Total & 100 & 100 & 100 & 100 & 100 & 100 \\
\hline $\begin{array}{l}\text { \% Promedio } \\
\text { gasto }\end{array}$ & 8,01 & 20,66 & 19,71 & 19,40 & 17,21 & 15,00 \\
\hline Total \% & 100 & 100 & 100 & 100 & 100 & 100 \\
\hline $\begin{array}{l}\text { \% s/ total } \\
\text { población }\end{array}$ & 8,0 & 20,7 & 19,7 & 19,4 & 17,2 & 15,0 \\
\hline
\end{tabular}

\section{Efecto de los gastos de salud en los ingresos}

El porcentaje de los principales gastos en dólares americanos (USD) que realizan los adultos mayores en Ecuador se han estructurado en la Tabla 10, con la finalidad de determinar el efecto de los gastos destinados a la salud, el resultado del análisis de la última encuesta muestra que, del promedio de ingreso total, que es de 470,31 USD, los adultos mayores destinan el 19,7 \% de sus recursos económicos a gastos médicos equivalentes en promedio mensual de 91,7 USD. Aplicando los modelos planteados para determinar el efecto que tiene el gasto de salud sobre los ingresos de los adultos mayores, se ha tomado en cuenta si los mismos requieren de hospitalización o no, los resultados evidencian que si es requerida la hospitalización la cantidad de dinero que deben destinar a los gastos médicos resultan muy superiores al promedio de ingreso total de la población adulta mayor, como se muestra en la Tabla 11. 
Tabla 10. Porcentaje de gastos de la población anciana (Datos tomados de la Encuesta SABE, 2017).

\begin{tabular}{|l|c|c|c|}
\hline \multicolumn{1}{|c|}{ Variables } & $\begin{array}{c}\text { Porcentaje } \\
\%\end{array}$ & $\begin{array}{c}\text { Ingreso mensual } \\
\text { USD }\end{array}$ & $\begin{array}{c}\text { Ingreso anual } \\
\text { USD }\end{array}$ \\
\hline Gastos de casa & 8,0 & 37,7 & 452,0 \\
\hline Gastos en comida & 20,7 & 97,2 & $1.166,2$ \\
\hline Gastos en ropa & 19,7 & 92,7 & $1.112,5$ \\
\hline Gastos médicos & 19.4 & 91.3 & 1.095 .0 \\
\hline Gastos de transporte & 17,2 & 81,0 & 971,4 \\
\hline Otros & 15,0 & 70,5 & 846,6 \\
\hline Ingreso total & 100 & 470,31 & $5.643,7$ \\
\hline
\end{tabular}

Tabla 11. Efecto del gasto en salud sobre los ingresos (Datos tomados de la Encuesta SABE, 2017).

\begin{tabular}{|l|c|c|}
\hline \multicolumn{1}{|c|}{ Variables } & Ingreso mensual & Ingreso anual \\
\hline Ingreso total (USD) & 470,31 & $5.643,74$ \\
\hline Costo de salud total Modelo 1 (con hospitalización) & $1.833,96$ & $22.007,54$ \\
\hline Costo de salud total Modelo 2 (sin hospitalización) & 190,39 & $2.284,68$ \\
\hline Diferencia Modelo 1 & $-1.363,65$ & $-16.363,80$ \\
\hline Diferencia Modelo 2 & 279,92 & $3.359,06$ \\
\hline Porcentaje de costo s/total ingreso Modelo 1 (\%) & 389,95 & 389,95 \\
\hline Porcentaje de costo s/total ingreso Modelo 2 (\%) & 59,52 & 59,52 \\
\hline № de veces Modelo 1 / modelo 2 & $1.032,36$ & 12,00 \\
\hline
\end{tabular}

\section{DISCUSIÓN}

El estudio sobre los costos en salud relacionados con los adultos mayores implicó considerar variables en función a las propuestas en los estudios realizados por autores como Santibáñez-Beltrán et al., (2013); Villarreal-Ríos et al., (2018); Sánchez-Román et al., (2012); Katon et al., (2003) y Quevedo-Tejero et al., (2011) comparando las variables y formas utilizadas para calcular el costo y efecto en los ingresos de los adultos mayores.

\section{Determinación de los costos de los servicios de salud para la población anciana}

Para la determinación del costo total de salud para el adulto mayor se consideró la fórmula 1. Los resultados muestran que el costo promedio mensual es de 190,39 USD y anual de 2.284,68 USD en el caso de no considerar costos de hospitalización (Modelo 2) y 1.833,96 USD por mes y 22.007,54 USD por año en el que se consideraron los costos de hospitalización (Modelo 1), como se especifica en la tabla 4. Por tanto, esta forma de determinar los costos permite aproximar los costos involucrados en los costos de salud desde la perspectiva del paciente, representados en el caso del modelo $1(389,95 \%)$ y en el modelo $2,(59,52 \%)$, lo que significa que el cálculo del costo de salud difiere del utilizado por Santibáñez-Beltrán et al., ( 2013); Villarreal-Ríos et al., (2018); Sánchez-Román et al., (2012); Katon et al., (2003) y Quevedo-Tejero et al., (2011), quienes consideran un solo recurso; es decir, un solo tipo de enfermedad, solo una consulta, atención de farmacia y medicación, como Santibáñez-Beltrán et al., (2013).

Por el contrario, en la presente investigación se consideran otros factores que, en el momento de una enfermedad en un adulto mayor, involucran gastos relacionados con la consulta médica, tratamiento médico, medicación, análisis de laboratorio y hospitalización si es necesario. Sin embargo, hubiera sido importante incluir en el cálculo del costo total, los costos de transporte que implica el traslado a un centro de salud privado para consultas médicas y a una farmacia para la adquisición de medicamentos, y a pesar de ello, representa un porcentaje considerable (17,2 \% s / Ingresos totales); ya que los costos de salud en adultos según los resultados de la investigación muestran los costos de salud privados en los que incurre una persona mayor, a pesar de que un porcentaje considerable cuenta con seguro público de salud. Así, desde este punto de vista, los costos influyen en los gastos de las personas mayores y estos a su vez en la disponibilidad de ingresos para otros gastos que no están relacionados con los gastos en salud. 


\section{Efecto de los gastos de salud en los ingresos}

Comparar los efectos de los costos con relación a los ingresos es otro aspecto analizado. Para este análisis se consideró lo que menciona Knaul et al., en 2018 "que los hogares afiliados a un sistema de salud tienen que incurrir en gastos de empobrecimiento". Los resultados de la investigación muestran que los costos en salud tienen un efecto negativo sobre los ingresos económicos de los adultos mayores, ya que es un factor de empobrecimiento, este fenómeno será mayor cuando se utilicen servicios de salud que no son financiados por el Estado sino por los ancianos. Se explica, porque en Ecuador, el gasto médico promedio representa el $19,4 \%$ del $100 \%$ del total de ingresos que recibe de diferentes fuentes, que en promedio es de 470,31 USD (Tabla. 10).

Sin embargo, si se consideran ambos modelos para determinar los costos, el gasto en el modelo 1 representa en promedio el 389,95\%, y en el caso del modelo 2 el 59,52 \%, siendo 9,63 veces superior al modelo 2, como se presentó en la Tabla11; lo que significa que un adulto mayor que no requiera hospitalización estaría por encima de la línea de empobrecimiento; en cambio en el caso de requerir hospitalización, los gastos de salud serían superiores a los ingresos de los ancianos; por lo que el efecto es negativo y el gasto en salud se convierte en un factor de empobrecimiento de esta población. Se demuestra que es cierta que la hipótesis H5 que planteó que los costos de salud se convierten en gastos de empobrecimiento para los adultos mayores.

En este sentido, los gastos en salud se convierten en gastos médicos de bolsillo para los ancianos, lo que afecta en gran medida los ingresos y el bienestar; tanto es así que alcanza los porcentajes de hogares que realizan gasto privado en salud en países desarrollados como Estados Unidos, Reino Unido, España, Francia, Holanda, Italia y Dinamarca, donde el gasto realizado por los hogares representó el $30 \%$ y el 8,4 \%, lo que significa que el gasto en salud de los adultos mayores de bajos ingresos representa una proporción mayor en relación a los de altos ingresos; que puede relacionarse con lo que la Organización Internacional del Trabajo y la Organización Panamericana de la Salud (OIT/OPS ) menciona en 1999, que : "el gasto que realizan las familias más pobres para la compra de bienes y servicios de salud representa una mayor proporción de lo que este gasto representa para las familias más ricas" (OIT/OPS, 1999), impactando en un empobrecimiento de esta población vulnerable y constituyendo un gasto catastrófico para los ancianos si fuera el caso del modelo 1 (costos de hospitalización 389,95 \%) y acercándose si fuera modelo 2 (sin gastos de hospitalización $59,52 \%$ ) ya que "se dice que un hogar tipo H sufre de gasto catastrófico en salud (GCSh) si los gastos que este tipo de hogar hace en salud directamente de su bolsillo son superiores al $30 \%$ de su capacidad de pago (CPh)" (Knaul et al., 2018).

Se evidencia que los costos privados son altos y que afectan las consultas médicas, tratamientos, hospitalización y medicación de los ancianos. Los servicios de salud subsidiados no cubren los servicios requeridos de manera oportuna, lo que provoca que los adultos mayores busquen otras alternativas, por lo que la mayoría de los adultos acude a los servicios privados. Se demuestra que: el costo de la atención, no problemas graves, tiempo de viaje, molestias típicas de la vejez, mala atención por parte de la familia, son factores causales del uso de los servicios de salud por parte de los ancianos; asimismo, el costo y la disponibilidad de medicamentos, no contar con transporte y la aversión a tomar medicamentos son factores que explican por qué la población anciana no toma la medicación prescrita para las distintas patologías que padece. Existe una relación significativa en los costos y el uso de los servicios de salud entre los centros de atención públicos y privados.

\section{CONCLUSIONES}

De acuerdo con los resultados obtenidos en este estudio, de su discusión y comparación con los resultados obtenidos por otros autores se pueden plantear las siguientes conclusiones principales:1. Se concluye que el costo relacionado con la polifarmacia es significativamente alto, seguido por los costos de hospitalización en relación con las consultas médicas y pruebas de laboratorio, considerando que aproximadamente un tercio de la población estudiada cuenta con seguro médico. 2. Los ingresos que tienen los adultos no cubren los costos de consulta, medicación, hospitalización y exámenes ya que la mayoría de los familiares cubren estos gastos. 3. Los gastos en salud son un factor de empobrecimiento de los ancianos, cuando son costos catastróficos, ya que los altos costos de salud influyen en los niveles de empobrecimiento cuando el anciano requiere hospitalización y las enfermedades pueden ser crónicas. 4. De acuerdo con los resultados las hipótesis planteadas en la presente investigación fueron contrastadas a través de la aplicación de la estadística multidimensional-regresión logística confirmando como ciertas todas las hipótesis.

\section{AGRADECIMIENTOS}

Esta investigación no recibió ninguna subvención específica de agencias de financiamiento del sector público, comercial o sin fines de lucro, sin embargo, la Universidad Nacional de Chimborazo (UNACH) permitió el uso de sus instalaciones y brindó la logística de transporte para los miembros del equipo de investigación, por lo cual el equipo de investigadores agradece a la UNACH el apoyo recibido. 


\section{NOTACIÓN}

$\mathrm{a}=$ Costos de medicación

$\mathrm{b}=$ Costos de hospitalización

$\mathrm{c}=$ Gastos de consulta médica

$\mathrm{d}=$ Costos de las pruebas de laboratorio

$\mathrm{C}=$ Costos directos de un recurso de salud utilizado

$\mathrm{Ca}=$ Costo total de los recursos sanitarios

i = Factorizar por el número de unidades obtenidas de cada recurso de salud

$\mathrm{Pi}=$ costo unitario del recurso de salud

$\mathrm{Qi}=$ Número de unidades de un recurso de salud dado utilizadas para la salud de los ancianos

\section{REFERENCIAS}

Arévalo-Avecillas, D., Game C., y otros dos autores, Predictores de la calidad de vida subjetiva en adultos mayores de zonas urbanas y rurales de la provincia del Guayas, Ecuador, http://dx.doi.org/10.4067/S0718-07642019000500271, Inf. tecnol., 30(5), 271-282 (2019)

Bail, K., Draper B., y otros tres autores, Predicting excess cost for older inpatients with clinical complexity: a retrospective cohort study examining cognition, comorbidities, and complications, https://doi.org/10.1371/journal.pone.0193319, PLoS One, 13(2), e0193319 (2018)

Bustamante, M.A., Lapo M.d.C., y otros dos autores, Factores socioeconómicos de la calidad de vida de los adultos mayores en la provincia de Guayas, Ecuador, https://doi:10.4067/S0718-07642017000500017, Inf. tecnol., 28(5), 165$176(2017)$

Bustamante, M.A., Vidal C.L., y López L.P., Impacto del uso de audífonos para adultos mayores en Chile, https://doi:10.4067/S0718-07642014000300020, Inf. tecnol., 25(3), 177-184 (2014a)

Bustamante, M.A., Vidal Silva C.L., y López L.P., Satisfacción de los adultos mayores por el uso de audífonos otorgados por el programa chileno de garantías explícitas en salud (GES), https://doi:10.4067/S0718-07642014000600019, Inf. tecnol., 25(6), 163-170 (2014b)

Caley, M., y Sidhu K., Estimating the future healthcare costs of an aging population in the UK: expansion of morbidity and the need for preventative care, https://doi.org/10.1093/pubmed/fdq044, J. Public Health, 33(1), 117-122 (2010)

Casas-Vásquez, P., Ortiz-Saavedra P., y Penny-Montenegro E., Estrategias para optimizar el manejo farmacológico en el adulto mayor, https://doi.org/10.17843/rpmesp.2016.332.2153, Rev Peru Med Exp Salud Publica, 33, 335-341 (2016)

Coory, M.D., Ageing and healthcare costs in Australia: a case of policy-based evidence? https://doi.org/10.5694/j.13265377.2004.tb06096.x , Med. J. Aust., 180(11), 581-583 (2004)

Cortés-Buelvas, A., Flor, E., y Duque, G., Análisis de costos de la atención médica hospitalaria. Experiencia en una clínica privada de nivel II-III, Colomb Med, 33(2), 45-51 (2002)

CELADE, Centro Latinoamericano y Caribeño de Demografía. Los adultos mayores en América Latina y el Caribe. Datos e indicadores. Madrid: Boletín informativo, edición especial con ocasión de la Il asamblea mundial de Naciones Unidas sobre el envejecimiento, 1-79, CELADE/CEPAL, Santiago de Chile, Chile, (2002)

Florence, C.S., Bergen G., y otros cuatro autores, Medical costs of fatal and nonfatal falls in older adults, https://doi.org/10.1111/jgs.15304, J. Am. Geriatr. Soc., 66(4), 693-698 (2018)

Gnanamanickam, E.S., Dyer S.M., y otros diez autores, Direct health and residential care costs of people living with dementia in Australian residential aged care, https://doi.org/10.1002/gps.4842, Int. J. Geriatr. Psychiatry, 33(7), 859-866 (2018)

González-González, C., Tysinger B., y otros dos autores, Projecting diabetes prevalence among Mexicans aged 50 years and older: The future elderly model-Mexico (FEM-Mexico), http://dx.doi.org/10.1136/bmjopen-2017-017330, BMJ Open, 7(10), e017330 (2017)

INEC, Instituto Nacional de Estadística y Censos (Ecuador), y CELADE (Organización), Ecuador, estimaciones y proyecciones de población, 1950-2025, Serie OI. № 205, 1-102, INEC/CELADE, Quito, Ecuador, (2003)

Katon, W.J., Lin E., y otros dos autores, Increased medical costs of a population-based sample of depressed elderly patients, https://doi:10.1001/archpsyc.60.9.897, Arch Gen Psychiatry, 60(9), 897-903 (2003)

Knaul, F.M., Arreola-Ornelas H., y otros tres autores, Efecto del seguro popular de salud sobre los gastos catastróficos y empobrecedores en México, 2004-2012, https://doi.org/10.21149/9064, Salud Publ Mex, 60(2), 130-140 (2018)

Li, C., Young B.R., y Jian W., Association of socioeconomic status with financial burden of disease among elderly patients with cardiovascular disease: evidence from the China health and retirement longitudinal survey, http://dx.doi.org/10.1136/bmjopen-2017-018703, BMJ Open, 8(3), e018703 (2018)

Marulanda-Castaño, O.J., Costos y presupuestos, 1-152, Universidad Nacional Abierta y a Distancia, Bogotá, Colombia (2009) 
Miller, T., y Mejía-Guevara, I., Personas adultas mayores: ensayos sobre sus derechos. El envejecimiento de la población en Ecuador: la revolución silenciosa, Consejo Nacional para la Igualdad Intergeneracional, 1-9, Quito, Ecuador (2020)

OIT/OPS. El gasto de Bolsillo en Salud en América Latina y el Caribe: Razones de Eficiencia para la Extensión de la Protección Social en Salud, Reunión regional tripartita de la ILO/OIT con la colaboración de la PAHO/OPS, 1-29, OIT/OPS, México, (1999)

Pilleron, S., Sarfati D., y otros cinco autores, Global cancer incidence in older adults, 2012 and 2035: a population-based study, https://doi.org/10.1002/ijc.31664, Int J Cancer, 144(1), 49-58 (2018)

Quevedo-Tejero, E.d.C., Zavala-González, M.A., y otros dos autores, Fractura de cadera en adultos mayores: prevalencia y costos en dos hospitales. Tabasco, México, 2009. Rev Peru Med Exp Salud Publica, 28(3), $440-445$ (2011)

Rechel, B., Grundy E., y otros cinco autores, Ageing in the European Union, https://doi.org/10.1016/S01406736(12)62087-X, Lancet, 381(9874), 1312-1322 (2013)

SABE, Encuesta de salud, bienestar del adulto mayor. Instituto Nacional de Estadística y Censos. (INEC) Quito, Ecuador, (2017)

Sánchez-Román, F.R., Carlos-Rivera, F.d.J., y otros cuatro autores, Costos de atención médica por cáncer cervicouterino, Rev. Med. Inst. Mex. Seguro Soc., 50(1), 99-106 (2012)

Santibáñez-Beltrán, S., Villareal-Ríos, E., y otros cuatro autores, Costo económico de la polifarmacia en el adulto mayor en el primer nivel de atención, Rev. Med. Inst. Mex. Seguro Soc., 51(2), 192-199 (2013)

Thavorn K, Maxwell C.J., y otros seis autores, Effect of socio-demographic factors on the association between multimorbidity and healthcare costs: a population-based, retrospective cohort study, http://dx.doi.org/10.1136/bmjopen2017-017264, BMJ Open 7(8), e017264 (2017)

Vargas-Aguilar, G.M., Olaya-Pincay, M.E., y otros dos autores, Incidencia de la valoración de enfermería en el autocuidado del adulto mayor, https://doi.org/10.47460/uct.v24i105.379, Universidad Ciencia y Tecnología, Vol. 24(105), 35-52 (2020)

Villarreal-Ríos, E., Julián-Hernández, Y.J., y otros cuatro autores, Costo de la atención médica en pacientes con enfermedad pulmonar obstructiva crónica, Rev. Med. Inst. Mex. Seguro Soc., 56(4), 371-378 (2018)

Wittenberg, R., Hu B., y otros siete autores, Projections of care for older people with dementia in England: 2015 to 2040 , https://doi.org/10.1093/ageing/afz154, Age Ageing, 49(2), 264-269 (2020) 